\title{
Wind-induced vibration piezoelectric energy collection in ventilation tunnels
}

\author{
Daiyong Zhou ${ }^{1,2, a}$, Yin Lin ${ }^{1,2}$, Gaojian Ren ${ }^{1,2}$ and Yan Shao ${ }^{1,2}$ \\ ${ }^{1}$ China Coal Technology Engineering Group Chongqing Research Institute, Measurement and Control Technology Research Branch, \\ Chongqing, China \\ ${ }^{2}$ State Key Laboratory of Gas Disaster Monitoring and Emergency Technology, Chongqing, China
}

\begin{abstract}
Ventilation tunnel wind-induced vibration piezoelectric energy collection uses MFC as piezoelectric material to make piezoelectric vibrators, and efficiently collects the wind-induced vibration energy in the ventilation tunnel and stores it in the energy storage device to provide the electrical energy required by the wireless sensor in the tunnel. According to the piezoelectric effect of piezoelectric materials, the instantaneous accumulated positive and negative charges generated at both ends of the piezoelectric vibrator at the instantaneous wind speed and wind vibration in the tunnel are collected. By establishing a piezoelectric energy collection model, the irregular transient charges are captured and stored as Available direct current. The piezoelectric energy harvesting model uses wind speed rotation as the traction force to drive the piezoelectric vibrator to vibrate, thereby converting wind energy into instantaneous electrical energy, and using the electrical energy harvesting device to store the electrical energy in the energy storage device. Experiments verify that when the wind-induced vibration piezoelectric energy collection model of the ventilation tunnel is at a wind speed of $8 \mathrm{~m} / \mathrm{s}$, the maximum output voltage of the energy storage device is $42.2 \mathrm{~V}$, which can meet the power supply requirements of wireless sensors in the ventilation tunnel.
\end{abstract}

\section{Introduction}

Ventilation tunnel wireless sensors play an important role in the entire coal mine safety monitoring system, because wireless sensor monitoring and monitoring has the advantages of rich interfaces, easy installation, avoiding hidden dangers and effectively reducing costs. Building a wireless sensor network in the harsh environment of a ventilation tunnel has become the basis for the construction of the physical layer of a smart mine. It is because building a sensor node network and wirelessly interacting information can realize real-time perception, monitoring and control of various information of the deployment area node ${ }^{[1-2]}$. Such as harmful gas concentration, temperature, wind speed and humidity. However, when considering the construction of wireless sensor network nodes, it is necessary to consider the power supply technology of wireless sensors.

Wireless sensor power supply technology is a key technology to ensure the normal operation and application development of wireless sensor networks, and it is also one of the key technologies for the construction of smart mines. Researchers have conducted research on wireless sensor network power supply technology by studying battery technology, energy harvesting technology and power intelligent control technology, and have achieved certain results. In terms of battery technology, some researchers have used pulsed laser deposition technology to prepare heterogeneous nanocomposite films and dianionic compound film materials as battery electrode materials, which can provide better electrochemical performance and provide a better energy storage system research direction. Some researchers have developed zincmanganese dioxide thin-film alkaline batteries, which are more environmentally friendly in terms of raw materials, are not sealed, have low cost, are flexible, can drive flexographic printed circuits, and have stable power supply capabilities ${ }^{[3-6]}$. The lithium thionyl chloride battery can be used as a backup battery, and can provide power for up to 10 years for wireless sensors with extremely small power consumption. In terms of energy harvesting technology, Millennial Net of the United States combines i-Bean wireless technology with the energy acquisition technology of the emerging company Ferro Solutions. The i-Bean wireless transmitter is driven by an inductive oscillation energy converter, which generates $28 \sim 30 \mathrm{~Hz}$ oscillation under the action of $50 \sim 100 \mathrm{mg}$. Converted to $1.2 \sim 3.6 \mathrm{mV}$ voltage value, which can achieve a distance of $30 \mathrm{~m}$ and send data at a rate of $115 \mathrm{~kb} / \mathrm{s}$. Piezoelectric MEMS MPG chip technology uses the end-loaded mass of a piezoelectric film to make a cantilever beam, and use external force to create dynamic vibration, capture the vibration energy after vibration, so as to realize the collection of micro energy. MEMS MPG chip energy harvesting technology has a power output range of $25 \sim 500 \mathrm{uW}{ }^{[7-10]}$. Japanese scholars have studied the vibration generated by the environment and machinery

\footnotetext{
a Corresponding author: zhoudaiyong123@163.com
} 
and equipment to convert electricity, and realize the automatic adjustment experiment of temperature and humidity, which greatly saves the operating cost of the system $^{[11-12]}$. In terms of power intelligent control technology, establish a variety of wireless network adaptive organizational structures based on the power consumption model of the system, study high-efficiency energy control algorithms to improve the networking protocol, and coordinate the configuration of the optimal energy ratio to achieve the optimal energy saving of wireless sensor networks mode. At present, the power supply intelligent control technology is still in the initial stage of development, and the new battery technology and energy harvesting power supply technology have become key research areas.

Ventilation tunnels are a special working environment in coal mines, which can be used as energy harvesting objects mainly including noise energy, electromagnetic energy, vibration energy and wind energy. Noise energy, electromagnetic energy and vibration energy all have continuous and reliable defects and are not suitable as natural resources for energy harvesting in ventilation tunnels. Wind energy is ubiquitous in the ventilation tunnel, providing a stable and reliable natural energy foundation for energy harvesting in the ventilation tunnel. In addition, piezoelectric materials have the conversion relationship between mechanical energy and electric energy, low cost, simple structure and easy processing. Therefore, exploring the relationship between wind energy and piezoelectric materials in ventilation tunnels can provide power supply technology for mine wireless sensors.

\section{Variation Law of Wind Energy in Ventilation Roadway}

The wind energy of the ventilation tunnel is the kinetic energy of the airflow in the tunnel, the formula for calculating the wind energy flowing through the crosssectional area $S$ perpendicular to the wind speed per unit time is

$$
W=1 / 2 S \rho V^{3}
$$

In the formula, $W$ is wind energy, the unit is $\mathrm{kg} \cdot \mathrm{m}^{2} \cdot \mathrm{s}^{-}$ ${ }^{3}, \rho$ is the ambient air density, the unit is $\mathrm{kg} / \mathrm{m}^{3}, V$ is the wind speed, the unit is $\mathrm{m} / \mathrm{s}$. It can be seen that the size of wind energy is directly proportional to the area through which the air flows, the air density and the cube of the air velocity. In the calculation of wind energy, the most important factor is the wind speed. The accuracy of the value of the wind speed has a decisive effect on the estimation of wind energy.

When the ventilation roadway is ventilated along the shaft, due to the viscosity and inertia of the air flow and the blocking and disturbing effect of the roadway wall facing the air flow, the ventilation resistance is generated, and different flow states are formed. When the flow velocity is low, the fluid particles do not mix with each other, and flow in a laminar direction parallel to the tube axis, which is called laminar flow, and the effect of wind speed is small. When the flow velocity is large, the size and direction of the fluid particles change at any time, and the fluid particles mix and flow in a turbulent manner, which is called turbulent flow, and the wind speed has a greater influence. Therefore, the distribution of wind energy in the ventilation tunnel is uneven. Through the study of the wind speed measurement and change law in the ventilation tunnel, it is found that the formula for calculating the wind speed in the turbulent core area of the tunnel is

$$
v=\frac{1}{k} \sqrt{\frac{\tau}{\rho}} \ln \mathrm{D}+C
$$

In the formula, $k$ is the mixing length coefficient, $\rho$ is the ambient air density, $\tau$ is the wall shear stress of the ventilation tunnel, $\mathrm{D}$ is the distance from a certain point of the tunnel to the wall, and $\mathrm{C}$ is the integral constant. The shear stress on the wall of the ventilation tunnel is

$$
\tau=\frac{1}{8} \lambda \rho \bar{v}^{2}
$$

According to the law of frictional resistance in turbulent flow

$$
\delta=\frac{\rho \lambda}{8}
$$

In the formula, $\delta$ is the friction resistance coefficient, $\lambda$ is the compensation coefficient, and $\bar{v}$ is the average wind speed at a certain point measured by the instrument. The instantaneous wind velocity can be calculated as

$$
v=\frac{1}{k} \sqrt{\frac{\delta \bar{v}^{2}}{\rho}} \ln \mathrm{D}+C
$$

It can be seen that under the condition of relatively stable air flow, the wall of the roadway is smooth and without any obstacles, and the distance between the change law of wind speed in the ventilation roadway and the wall of the roadway shows a logarithmic law. Therefore, according to the calculation formula of wind speed and wind energy, the amount of wind energy in the ventilation tunnel is affected by the wall and obstacles, and the distance from the tunnel wall has a logarithmic relationship.

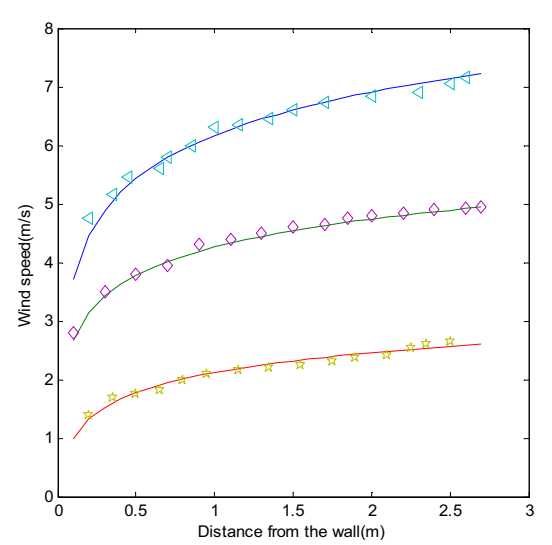

Fig.1 The relationship between instantaneous wind speed and roadway wall distance 


\section{Piezoelectric energy harvesting device}

The piezoelectric energy collection device converts wind energy in the ventilation tunnel into vibration energy, and uses piezoelectric materials as the energy conversion medium to collect wind-induced vibration energy. The piezoelectric energy collection device converts wind energy in the ventilation tunnel into vibration energy, and uses piezoelectric materials as the energy conversion medium to collect wind-induced vibration energy. The piezoelectric materials commonly used for energy harvesting mainly include piezoelectric ceramics PZT, piezoelectric polymer PVDF and piezoelectric fiber composite material MFC. Various materials have advantages and disadvantages, due to the particularity of Ventilation environment need to be considered flexible piezoelectric material, lightweight, low cost and high sensitivity, the piezoelectric material more suitable choice of MFC. It is composed of rectangular piezoelectric ceramic rods sandwiched between adhesives, electrodes and polyimide film layers. The electrodes are attached to the film in a cross pattern. The cross electrodes will output voltage from the strip piezoelectric rods. Induction of deformation, noise and vibration, it is an excellent material for obtaining energy from vibration. The characteristics of MFC piezoelectric materials are not only the properties of dielectric, but also the elastic medium, which is a special material with piezoelectric body interchangeability. The electrical parameters and mechanical parameters describe the laws of piezoelectric materials as ${ }^{[13-16]}$

$$
Q=T \mathrm{~d}+\varepsilon E
$$

Where $Q$ is the potential difference, $T$ is the piezoelectric stress, $\mathrm{d}$ is the piezoelectric constant matrix, $\varepsilon$ is the electrical coefficient matrix of the piezoelectric material under constant stress, and $E$ is the electric field. In the absence of an external electric field, the piezoelectric material is only affected by the $T$ stress. The free end of the piezoelectric vibrator can be freely deformed after being excited by the stress, resulting in a potential difference between the two ends. The piezoelectric internal resistance is much greater than the load resistance. The charge is transferred to the external circuit. Through analysis, it can be known that the magnitude of the dynamic potential difference is related to the deformation of the piezoelectric layer, and the deformation of the piezoelectric layer is related to the piezoelectric material and the wind energy of the ventilation tunnel. The piezoelectric energy harvesting device uses a rotating body structure to drive multiple piezoelectric sheets to perform mechanical vibration motion, which increases the frequency and amplitude of the deformation of piezoelectric materials, and can efficiently convert wind energy into dynamic AC electrical energy. The structure of the energy harvesting device is

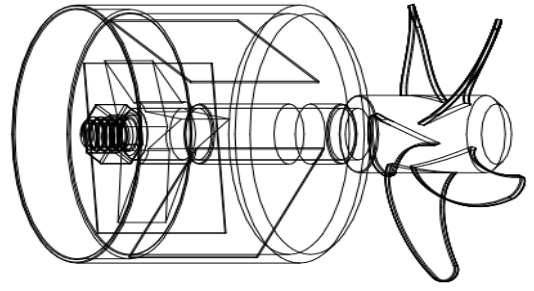

Fig.2 Mechanical vibration structure of piezoelectric energy harvesting device

The instantaneous voltage values that can be measured for the rotating body structure of the piezoelectric energy harvesting device under different wind speeds are shown in Figure 3.

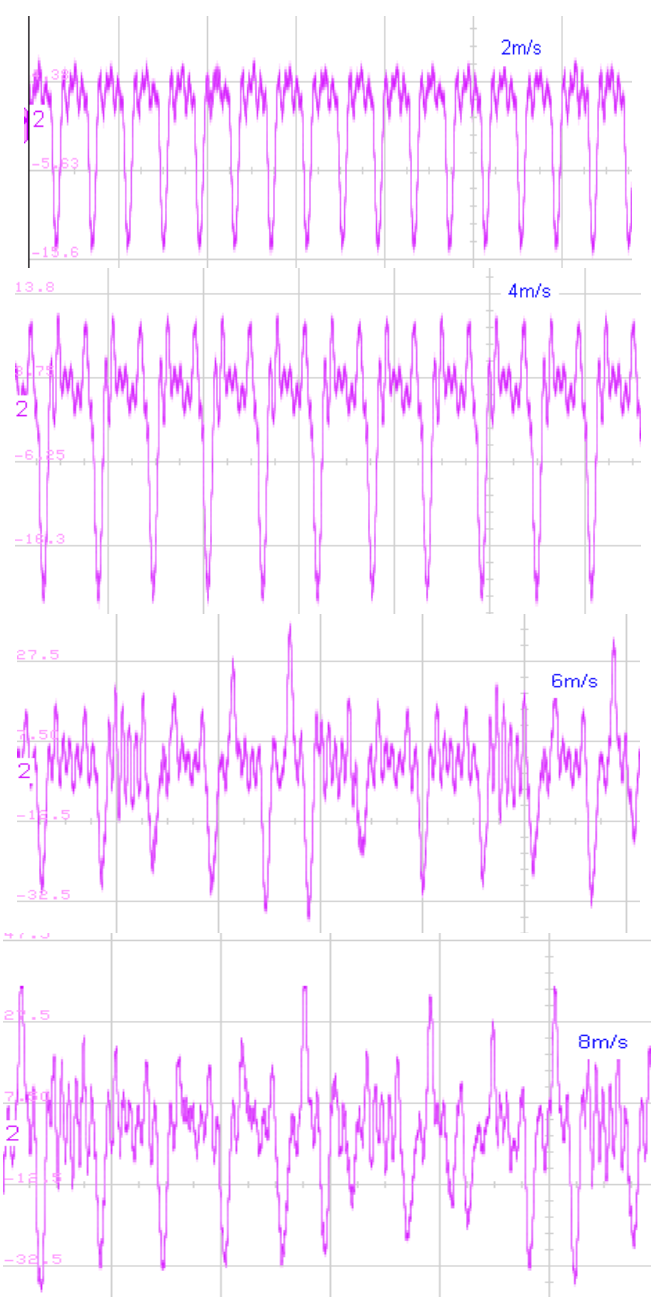

Fig.3 Piezoelectric instantaneous voltage and wind speed

\section{Energy harvesting management and output}

According to the electrical energy collected by the piezoelectric energy harvesting device, the electrical energy at both ends of the piezoelectric material is irregular and unstable AC output, which cannot be directly supplied to the wireless sensor. The collected piezoelectric electrical energy needs to be converted into the voltage required by the wireless sensor. The power circuit for collecting energy management includes a rectifier circuit, 
a filter circuit, a voltage regulating circuit, an energy storage circuit, and a voltage stabilizing circuit. The collected piezoelectric alternating current is converted into storable direct current, and each module circuit is independently designed. Not only the design circuit is complicated, but also the online energy loss is large, and the available effective energy is small. Therefore, considering the use of micro-energy harvesting and management power chips to integrate full-wave bridge rectifiers and high-efficiency step-down converters, the input voltage range can be expanded to between $2.7 \mathrm{~V}$ and $50.0 \mathrm{~V}$, and the output voltage can be configured as an adjustable voltage. Many wireless sensors provide power.

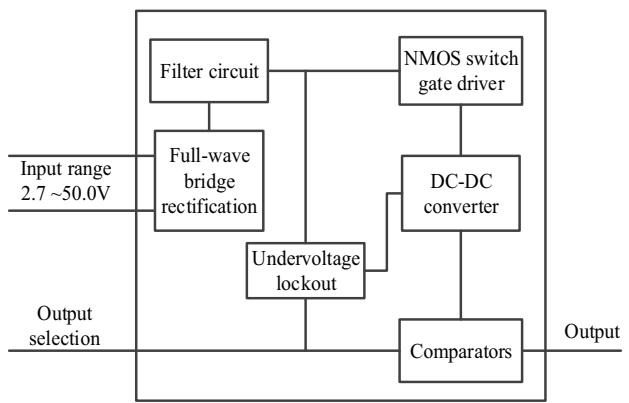

Fig.4 Block diagram of piezoelectric energy harvesting circuit

The wind-induced vibration piezoelectric energy collection model device for ventilation tunnels collects and stores the continuous and uniform wind energy in the ventilation tunnels in related energy storage devices, and supplies power to the wireless sensors in the tunnels. According to relevant regulations, the maximum wind speed in the ventilation tunnels is $8 \mathrm{~m} / \mathrm{s}$, in the range of $8 \mathrm{~m} / \mathrm{s}$ wind speed, the output voltage is shown in Figure 5.

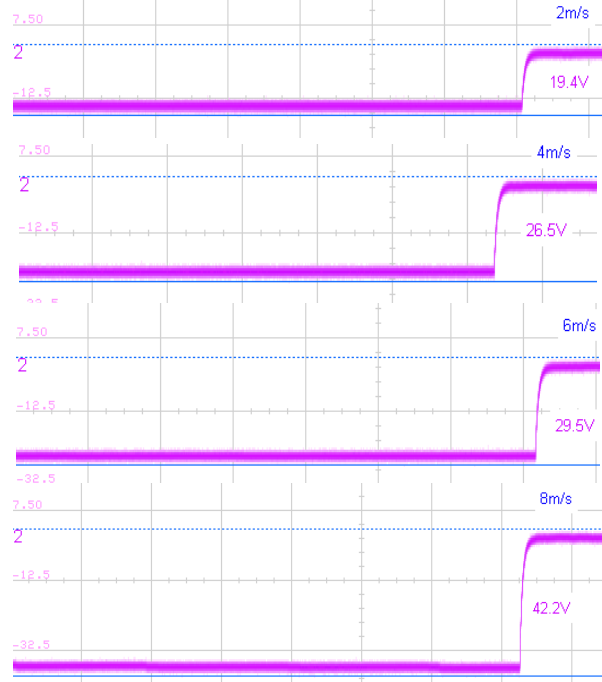

Fig.5 Output voltage and wind speed

\section{Conclusion}

Wind-induced vibration piezoelectric energy collection for ventilation tunnels uses MFC as a piezoelectric material to make piezoelectric vibrators. The instantaneous accumulated positive and negative charges generated at the two ends of the instantaneous wind speed and wind vibration piezoelectric vibrators in the tunnel are collected. By establishing a piezoelectric energy collection model, the irregular transient charge is captured and stored as usable direct current to power the wireless sensors in the tunnel. Through experimental verification, when the wind speed is $8.00 \mathrm{~m} / \mathrm{s}$, the maximum output voltage of the energy storage device is $42.2 \mathrm{~V}$, which can meet the power supply requirements of wireless sensors in the ventilation tunnel.

\section{Acknowledgments}

This article is based on Special Fund Project for Scientific and Technological Innovation and Entrepreneurship of Tiandi Science \& Technology Co., Ltd. "Research on Underground Wind-Induced Vibration Piezoelectric Energy Harvesting Technology" (Project No.: 2020-TDQN012) and "Distributed Underground Coal Mine One of the phased achievements of Research on Power Supply Technology (2020-TD-MS013).

\section{References}

1. Y. Chen, J.L. Zhou, Y.CH Wu. Design of Mine Flameproof Wind Power Supply Device.Coal Mine Machinery,38,06( 2017) .

2. Q.J. Xu, L.L. Ge,CH.H. Zong, Design of SelfPowered Power Supply of Sensor for Piezoelectric Energy Harvesting Piezoelectrics\& Acoustooptics, 41, 02,(2019)

3. X.ZH. Du, L.B. ZHang, H.Yu, Research Status of the Self-Powered Sensor Energy Harvesting Technology. Micronanoelectronic Technology,55,04,(2018)

4. S. Song, Y. Hou, M. Guo. An investigation on the aggregate-shape embedded piezoelectric sensor for civil infrastructure health monitoring. Construction and Building Materials, , 131,(2017)

5. X. Shi, X. Zhang, Q. Yaol. A novel method for the rapid detection of microbes in blood using pleurocidin antimicrobial peptide functionalized piezoelectric sensor. Journal of Microbiological Methods, 133,(2017)

6. J. Dong, Z. Wang, X. Kang. The synthesis of graphene/PVDF composite binder and its application in high performance $\mathrm{MnO} 2$ supercapacitors. Colloids and Surfaces, Physicochemical and Engineering Aspects, 489,(2016)

7. M. ZHang,Y.L. Xie, L. Lei.Numerical Research of Piezoelectric Energy Harvesting from VIV Based on XFlow.Journal of Chongqing Jiaotong University (Natural Science) ,36,01,( 2017)

8. SH.SH. Cai, J.H. Yang.Analysis of Piezoelectric Technology and its Applications on Road Energy Collection.Industrial Technology Innovation, 16,05, (2016)

9. H. Wang,W.Q. Qiu , X. Zhou. A Multiple Mass Broadband Piezoelectric Energy Harvesting Device. Piezoelectrics \& Acoustooptics,37,06, (2015)

10. F.X. Qu,Y.SH. Xia, G. SHi.Optimized Design of 
Self-Powered Synchronous Charge Extraction Circuit.Chinese Journal of Sensors and Actuators, 29,03, (2016)

11. ZH.M. CHen, X. Rong, G.ZH. Cao.Design of a selfpowered power based on piezoelectric energy harvesting technology.Electronic Design Engineering, 24,10,(2016)

12. D.B. Wang,ZH. Su, ZH.CH. SHi.Research on Piezoelectric Energy Harvesting of Variable Triangular Cross-sections Based on Equivalen. Chinese Journal of Solid Mechanics, 40,05, (2019)

13. H.J. Zou, Z.P. Wang, J. Song.Piezoelect ric Energy Harvesting Based on Nozzle-resonator System. Science \& Technology Vision, 229,07, (2018)

14. Y.H. Zhao, J.R. Liang, K. ZHao. Design and implementation of a self-powered piezoelectric energy harvesting system. Electronic Design Engineering, 379,05, (2018)

15. N.R. Alluri, S. Selvarajan, A. Chandrasekhar. Piezoelectric BaTiO 3/alginate sphericalcomposite beads for energy harvesting and self-powered wearable flexion sensor. Composites Science and Technology, 142,( 2017)

16. N. Chen, H. J. Jung, H. Jabbar. A piezoelectric impact-induced vibration cantilever energy harvester from speed bump with a low-power power management circuit. Sensors and Actuators A: Physical, 254,(2017) 